\title{
Strates
}

STRATES Matériaux pour la recherche en sciences sociales

Hors-série | 2002

Parcours dans la recherche urbaine, Michel Rochefort, un géographe engagé

\section{Regard sur la recherche urbaine}

avancées et perspectives

Jacques Brun et Catherine Paix

\section{(2) OpenEdition}

Journals

Édition électronique

URL : http://journals.openedition.org/strates/557

DOI : $10.4000 /$ strates.557

ISSN : $1777-5442$

Éditeur

Laboratoire Ladyss

Édition imprimée

Date de publication : 1 janvier 2002

ISSN : 0768-8067

\section{Référence électronique}

Jacques Brun et Catherine Paix, « Regard sur la recherche urbaine », Strates [En ligne], Hors-série |

2002, mis en ligne le 17 mai 2005, consulté le 08 septembre 2020. URL : http://

journals.openedition.org/strates/557 ; DOI : https://doi.org/10.4000/strates.557

Ce document a été généré automatiquement le 8 septembre 2020.

Tous droits réservés 


\title{
Regard sur la recherche urbaine
}

\author{
avancées et perspectives
}

Jacques Brun et Catherine Paix

Du côté des géographes : Quels approfondissements?

1 Tu es l'un des acteurs, et l'un des témoins privilégiés, du développement de la recherche urbaine et de ses évolutions dans les trente dernières années. Quelles ont été selon toi les principales avancées de la géographie dans ce domaine? Avec le recul, quel bilan peux-tu faire de la place et du rôle des géographes dans ces évolutions?

2 Il me semble que les géographes ont beaucoup apporté dans l'analyse et la compréhension de la diversité urbaine. Dans les années soixante-dix, la sociologie urbaine a connu un très grand essor qui a un peu mis à l'écart les efforts des géographes, mais par la suite il y a eu une certaine «réconciliation », c'est-à-dire un effort pour assumer l'apport de chacun. Et, plus je lis, plus je pense que l'approche des géographes dans le domaine urbain s'est enrichie, qu'elle foisonne d'analyses très intéressantes, mais que l'on est aujourd'hui dans une phase où les recherches sont tellement diverses, sur le plan théorique comme au niveau de la découverte de la réalité, que l'on manque de synthèses. Je viens par exemple de lire des études passionnantes de jeunes chercheurs qui travaillent avec l'équipe d'Alain Dubresson et Sylvy Jaglin ${ }^{1}$ sur l'Afrique du Sud depuis la fin de l'apartheid. C'est l'exemple même d'un travail riche, que j'ai été content de découvrir. Mais, plus généralement, je pense qu'avec des hauts et des bas, la géographie urbaine a tout doucement progressé, depuis vingt ans, et qu'elle continue à progresser parce qu'elle repose de plus en plus sur une approche socio-spatiale qui ne sépare plus, dans l'analyse, la dynamique socioéconomique du groupe humain de ses rapports avec l'espace.

3 À travers la thèse de Pascale Froment sur l'économie souterraine de Naples, on voit exactement ce que peut être la géographie d'aujourd'hui ${ }^{2}$. Elle fait une analyse en profondeur de la réalité sociologique et économique des petites entreprises illégales de Naples, et en même temps elle montre que ce secteur n'a pu se développer et ne peut survivre que parce qu'il s'agit du centre de Naples. C'est le centre historique dans cette conjonction sociale, économique et spatiale particulière, qui est l'élément qui fait que ça marche. Depuis des années, quand quelqu'un me parle de son sujet de thèse et me dit 
"ce n'est peut-être pas de la géographie ", je lui réponds "si vous analysez les phénomènes sociaux dans leurs relations avec l'espace, cela m'intéresse de diriger votre thèse ». Je pense que nous en sommes là. Il y a aujourd'hui une approche pluridisciplinaire des problèmes urbains, car tout le monde ressent plus ou moins la nécessité de tenir compte de tous les facteurs et de leurs interactions.

4 Dans les grands mouvements de la recherche urbaine des trente dernières années, quelles sont les inflexions, les avancées qui te semblent avoir été les plus importantes? Comment te positionnes-tu par exemple par rapport aux approches quantitativistes?

5 Je ne domine pas assez tout ce qui a été écrit sur l'urbain pour répondre complètement à la question. Il nous faudrait avoir plus de réflexions de synthèse sur le sujet pour dégager ces inflexions. En ce qui concerne le mouvement quantitativiste, je pense qu'à ses débuts il a permis d'améliorer un certain nombre d'études mais qu'il est devenu de plus en plus une entrave aux progrès de la recherche urbaine. En France, il y a eu un moment où j'ai trouvé cette direction intéressante, même si elle me dépassait sur le plan technique. Je pense qu'en tant qu'outil cela a permis d'aller plus loin. Maintenant, il me semble que ceux qui en sont restés là s'enferment. Et, ce n'est certainement pas un hasard si les services de financement de la recherche, ou certains services d'aménagement comme la Datar font appel à eux parce que cela évite de poser des problèmes sociaux trop graves. De mon point de vue, c'est donc plutôt une régression. Les Américains s'étaient d'ailleurs aperçus bien avant nous des dangers auxquels pouvaient conduire une telle démarche. Il me semble avoir lu il y a une vingtaine d'années une autocritique de Brian Berry, à la suite de son étude sur les systèmes urbains chiliens qui était, il faut bien le dire, particulièrement dénuée de bon sens. L'absurdité, la caricature, c'est ce qu'ont fait de l'analyse quantitative à la Berry les Brésiliens qui avaient opté pour la dictature! Ils ne voulaient plus sortir de leur cabinet, et surtout pas faire d'études de terrain, car toute étude de terrain était perçue comme un danger puisque suspectée de participation aux luttes sociales. Pendant la dictature, tous mes amis ont été mis un peu à l'écart, ou se sont exilés comme Milton Santos, et au Conseil national de Géographie où j'avais travaillé quelques années, ce sont les quantitativistes de cabinet qui ont pris le pouvoir, en copiant aveuglément les analyses factorielles et autres de Brian Berry. J'ai vu alors le système urbain de Pernambouc analysé avec des indicateurs qui n'avaient aucun rapport avec la réalité brésilienne parce qu'ils avaient été repris de Berry.

6 Pour ce qui est du reste, dans les trente dernières années, je ne parlerais pas tellement d'inflexion. Je dirais plutôt qu'il y a eu approfondissement d'une démarche que certains appellent socio-spatiale et sur laquelle un grand nombre de chercheurs se sont mis d'accord dans les années quatre-vingt. Il y a un approfondissement de ce qui me paraît être la démarche juste, et cela a certainement été facilité par de nouveaux outils, y compris l'informatique. Mais prendre l'outil pour la démarche, je considère que c'est une régression par rapport aux avancées actuelles des approches dans le domaine urbain.

Un lent rapprochement de la géographie et de la sociologie

$7 \quad T u$ as toujours œuvré pour encourager la pluridisciplinarité et tu n'as cessé de dialoguer avec des chercheurs d'autres disciplines, économistes et sociologues notamment. Dans un précédent entretien tu nous a dit que les travaux des sociologues t'avaient interpellé, mais que leur approche de la question urbaine te semblait parfois un peu trop dogmatique, qu'ils te semblaient "s'enfermer dans un vocabulaire dans lequel tu avais beaucoup de mal à entrer... ». Il y a par 
ailleurs des chercheurs qui se réclament d'un certain marxisme, et qui ont trouvé chez toi un appui, une capacité de dialogue pour éclaircir leurs propres positions qui leur a été très utile. Cela a tout particulièrement été le cas dans le cadre du Laboratoire de géographie humaine que tu as longtemps dirigé. Sur quels points, plus précisément, portaient tes désaccords par rapport à la sociologie marxiste des années soixante-dix?

On peut admettre qu'il y avait un problème de compréhension entre deux disciplines différentes. Chacune avait son langage, et celui des sociologues me paraissait un peu obscur. Mais sur le fond, ce qui m'a le plus gêné chez eux, c'est le systématisme de leurs explications, même si j'étais impressionné par leur bagage théorique. Dans leurs analyses on retrouvait, presque toujours, les structures sociales, la lutte des classes, et finalement la "crise urbaine ». Mais ils ne tenaient pas compte de la spécificité des situations locales à différentes échelles, ni de l'influence des formes héritées d'organisation de l'espace dans les modes de déroulement de ce qu'ils appelaient la crise urbaine. Autrement dit, j'avais une réaction - sans doute discutable - de géographe. J'avais l'impression qu'à force de vouloir théoriser ils passaient à côté de la richesse de la réalité urbaine c'est-à-dire de l'intensité variable et de la diversité des formes de la crise urbaine. C'était une question sensible pour moi, parce que j'avais oublié cette diversité au moment où je travaillais sur les métropoles d'équilibre. On en revient à cet effort qui a été le mien entre 1970 et 1980 pour essayer d'appréhender les différences sans les dissocier des mécanismes plus globaux d'explication, que ce soit à l'échelle du monde développé ou en développement, à l'échelle des pays européens et de la France ou à celle des différents types de villes. Je me souviens en particulier de ce fameux colloque de Dieppe, où j'avais été très séduit par l'analyse d'Henri Coing, qui s'inscrivait à ce moment là dans la lignée des sociologues marxistes, et en même temps un peu décontenancé par la vigueur de ses modèles d'interprétation globale. Les généralisations des sociologues me mettaient mal à l'aise, au regard de l'extrême diversité des situations, même si cette diversité était effectivement secondaire par rapport aux principaux facteurs d'explication qu'ils mettaient en avant. Pour ma part, je me situais quand même toujours dans la perspective de l'aménagement, et je pensais que cela ne servait à rien de dénoncer le système capitaliste et ses effets - même si leurs analyses au niveau global étaient exactes -, si on n'avait pas une idée des différences sur lesquelles fonder une éventuelle démarche d'aménagement. Le capitalisme est un fait, les inégalités socio-spatiales aussi. Mais il peut y avoir une certaine marge de manœuvre en matière d'aménagement, et cette marge de manœuvre passe par la compréhension des différences. On sait bien que l'on ne va pas résoudre la question des inégalités, mais on peut apporter certaines améliorations à des situations existantes, qui seront variables selon les contextes. Telle était en gros ma position dans le débat des années soixante-dix entre géographes et sociologues, d'autant que mes rencontres avec les sociologues se faisaient souvent par le biais de réflexions sur l'aménagement urbain.

Dans les années quatre-vingt, je pense que les sociologues ont évolué, du moins ceux que je connais. Quand je présidais la Commission de financement de la recherche sur les pays en développement au ministère de la Recherche, c'était en 1982, certains plaidaient toujours pour que toute proposition de programme soit fondée sur une base théorique indispensable mais qu'elle soit assortie aussi d'études de cas précis permettant d'étayer les hypothèses de départ et de saisir les nuances et la richesse de la réalité. Par rapport à d'autres qui étaient peut-être plus enclins à des approfondissements théoriques, ils insistaient toujours sur la nécessité de faire des 
études de cas. Telles étaient un peu les tendances. Je pense que ceux que j'ai connus et avec lesquels j'ai travaillé dans les années 1970-80 se sont ensuite tout à fait rapprochés de ce qui était mon point de vue, peut-être en partie parce qu'ils étaient dans des commissions, où, après 1981, il y avait un petit espoir de faire quelque chose.

10 En tant que géographe tu insistes sur la nécessité de comprendre la réalité urbaine dans sa dynamique globale et sa diversité. Dans la conception qui est la tienne, y a-t-il des éléments ou des facteurs de différenciation qui te semblent plus importants que d'autres, et dont la prise en compte aurait sans doute permis aux sociologues de progresser davantage dans l'application de leurs théories?

11 C'est une question à laquelle je ne peux pas répondre, car tout dépend des échelles. À l'échelle du monde, on ne peut pas plaquer sur la question urbaine une théorie d'explication générale, sans tenir compte fondamentalement du fait que les réalités sont très différentes selon qu'il s'agit de sociétés qui ont été colonisées, dominées, ou qui continuent à être dominées, et de sociétés dont le système économique a reposé sur la domination. À l'échelle des pays, qu'ils soient du Nord ou du Sud, ce sont les héritages historiques, c'est-à-dire le poids des formes héritées d'organisation sociospatiale, qui constituent sans doute les principaux facteurs de différenciation. Dans les pays du Sud, par exemple, les situations ne sont pas du tout les mêmes là où la ville a été générée par la domination, - c'est-à-dire dans des espaces qui n'étaient pratiquement pas urbanisés -, et là où d'anciennes formes et traditions urbaines ont été réutilisées. Pour interpréter ces différences, il faut donc revenir sur les différentes périodes auxquelles s'est exercée la domination et sur ses formes variables selon les espaces. Quand l'échelle d'analyse devient plus grande, les facteurs qui entrent en jeu dans la diversité des situations sont plus nombreux ; ils sont aussi très variables selon les cas. Je pense par exemple, qu'une des choses que je n'ai pas analysées dans ma thèse sur l'organisation urbaine de l'Alsace, et qui contribue à différencier cette région par rapport à d'autres régions françaises, c'est l'importance du phénomène culturel et religieux. Enfin, à l'échelle de chaque ville, c'est le dynamisme social et économique, en particulier de la bourgeoisie, qu'il faut probablement privilégier, car c'est ce qui fait marcher une ville et ce qui contribue à différencier les villes entre elles. Dit brièvement, ce sont là, aux différentes échelles, quelques éléments fondamentaux qui vont nuancer et différencier la simple projection des rapports sociaux dans l'espace, dont l'analyse n'est pas suffisante pour expliquer les différences.

12 Dans ce qui t'a distancié par rapport aux sociologues et dans ta démarche, est-ce qu'il ne s'agissait pas pour toi de pousser en fait plus loin l'analyse marxiste? Dans un entretien, tu as dit que tu te situais plus du côté d'une "géographie applicable " que d'une "géographie active " contestataire, est-ce que tout en prenant cette position tu penses avoir gardé une démarche marxiste?

À condition d'envisager le marxisme d'une façon très large, c'est-à-dire comme l'analyse de l'influence des structures sociales et donc des structures inégalitaires des sociétés capitalistes sur l'organisation de l'espace. À ce moment-là, oui, certainement.

Mes capacités à m'affirmer ou à m'engager ont été variables. À certains moments je me suis senti en porte-à-faux par rapport aux analyses trop théoriques de certains marxistes ; à d'autres, j'ai eu l'impression que cela devenait tellement vain de continuer à faire des analyses pour l'aménagement que j'ai un peu baissé les bras. Mais, sur le fond, je ne pense pas avoir changé. 
15 À l'époque de ma thèse j'ai fait du marxisme un peu sans le savoir parce que j'étais beaucoup plus un homme de terrain, et que je n'étais pas très formé à la théorie marxiste. Il s'est trouvé que je me suis intéressé aux rapports entre structures sociales et formes d'organisation de l'espace et que j'ai insisté là-dessus, ce qui m'a conduit à mettre l'accent sur le rôle des bourgeoisies dans l'organisation de l'espace, mais j'y suis arrivé selon une démarche qui était très empirique. Après, il y a eu une parenthèse pendant laquelle j'ai été pris dans cette espèce de machine à broyer qu'est l'aménagement du territoire officiel. Cela a été ma période fonctionnaliste, celle des métropoles d'équilibre. Mais par la suite, j'ai retrouvé cette volonté, ce souci, ce besoin de relier structures sociales et organisation de l'espace et cela ne m'a plus quitté. Cela a été plus affirmé dans ce que j'ai pu faire au niveau du financement ou de la direction de la recherche que dans ce que j'ai écrit, parce que j'ai assez peu écrit, mais je ne pense pas avoir beaucoup changé. Il y a donc eu plus ou moins d'engagement de ma part, mais la ligne générale de ma démarche n'a pas changé.

Quelles nouvelles directions pour la géographie urbaine aujourd'hui ?

16 Dans quelles directions penses-tu qu'il faudrait orienter les travaux des jeunes chercheurs? Qu'est ce que l'on devrait en attendre selon toi?

Le jeune chercheur, lui, doit prendre une direction nouvelle qui est nécessairement assez pointue, et pour l'instant, ce que la recherche des jeunes apporte, c'est d'être très proche du terrain. Les Brésiliens par exemple, sont très attirés pour l'instant par le problème de la participation populaire à l'aménagement . Or, il ne sert à rien d'étudier cette participation à l'échelle du Brésil. Si on veut aller plus loin, il faut essayer de voir que le problème ne se pose pas toujours de la même façon selon les villes. Lorsque l'on prend des cas précis de participation, soutenus par les collectivités locales, des ONG, etc., on voit par exemple qu'à Recife, ces problèmes-là ne se posent pas du tout dans les mêmes termes qu'à Fortaleza, alors que ce sont deux villes du Nord-Est. Mais elles n'ont pas connu la même histoire et le contenu des forces sociales y est très différent. Ce qui est intéressant c'est donc d'essayer de savoir, - au-delà de tout ce que l'on peut raconter sur les stigmates de la pauvreté - , pourquoi cela marche à Recife, et pourquoi dans les conditions qui sont celles de Fortaleza cela ne marche pas, et de montrer que les mécanismes que l'on observe à Recife ne sont pas transposables à Fortaleza et réciproquement. Quelle est la spécificité de ces populations pauvres? Comment expliquer que leurs réactions soient différentes? C'est ce genre de questions que les thésards peuvent creuser. On a maintenant sur les problèmes urbains une masse d'idées générales, et ce qu'il faut donc connaître pour pouvoir avancer ce sont les mécanismes qui s'accrochent à une réalité très locale. Ceci étant, on a de plus en plus d'études, et ce qui me gêne le plus actuellement c'est que l'on manque de synthèses. La synthèse est une démarche qui demande évidemment d'être relativement âgé pour arriver à aller plus loin dans les grandes idées générales en tenant compte de tout ce qui a été fait, mais on peut aussi y inciter les thésards lorsqu'ils ont la formation ou l'état d'esprit pour le faire. C'est une chose dont je rêvais et qui finalement n'a jamais été faite sous ma direction, mais j'aimerais bien que d'autres le fassent :demander à un thésard de faire une "thèse des thèses " sur un sujet donné, c'est-à-dire dégager d'un certain nombre de thèses ce qui fait avancer la réflexion générale. Cela me semble nécessaire, car il y a un trop grand hiatus entre ce que l'on sait au niveau général et les richesses contenues dans un certain nombre de thèses qui n'ont jamais été publiées et qui n'ont jamais été vraiment utilisées. Finalement cela n'a jamais été fait sous ma direction, 
mais j'aimerais bien que d'autres dirigent ce genre de travaux. Moi-même, je pense que j'ai perdu énormément de la richesse que pouvaient m'apporter toutes les thèses que j'ai dirigées. Je m'en suis servi pour mes cours, cela me donnait des idées, mais je n'ai jamais fait cet effort plus global de synthèse. C'est dans ces deux directions qu'il faudrait selon moi orienter les jeunes chercheurs.

Dans ce processus d'approfondissement - et donc pas d'inflexion de la recherche urbaine -, que penses-tu de l'importance croissante qui est accordée, à la fois sur le plan problématique et méthodologique à la démarche anthropologique? Est-ce qu'il te semble que dans l'approfondissement d'une démarche socio-spatiale, et compte tenu de la problématique urbaine actuelle, il est nécessaire de redescendre jusqu'au niveau de l'individu? Est-ce que cela ne risque pas de nous éloigner de notre objet, ou au pire de conduire à une impasse comme cela a été le cas de la démarche quantitative dont tu viens de parler?

C'est pour moi un vrai problème. Je pense qu'une démarche de type anthropologique peut-être utile, voire nécessaire, pour aller plus loin dans la compréhension des mécanismes de différenciation socio-spatiale, à condition de ne pas perdre de vue que ce que l'on cherche à comprendre, en tant que géographes, ce sont beaucoup plus les interrelations entre groupes sociaux et territoires que les relations entre les individus eux-mêmes, et à condition de ne pas s'enfermer dans l'analyse des comportements individuels. Certaines études qui ont été dans ce sens, ne me semblent pas entièrement satisfaisantes.

Une des questions que nous avons à creuser c'est le rôle actif de l'organisation de l'espace, ou en d'autres termes de l'espace social déjà constitué, dans la dynamique même des forces sociales. Je suis donc tout à fait convaincu que les phénomènes d'identification socio-spatiale, qui ont conduit à insister sur l'espace vécu, ou sur les comportements individuels ou familiaux d'insertion urbaine des migrants méritent d'être approfondis. Cette approche m'a parue très intéressante à ses débuts, et j'ai moimême engagé beaucoup de jeunes dans cette direction. Maintenant, je suis plus réservé quant aux résultats, car il y a une certaine «dérive anthropologique " qui risque d'aboutir à des résultats peu synthétisables et qui peut conduire à une impasse par rapport à nos questionnements plus généraux. Ceci dit, je pense qu'il est nécessaire de redescendre jusqu'à l'acteur individuel pour aller au bout de la compréhension de la relation d'un groupe social avec son espace, à condition de le mettre à sa place, de bien resituer les conditions d'individualisation de son action dans leur contexte, c'est-à-dire dans le cadre des relations de groupe, et de la réalité sociale et économique plus globale dans laquelle elle s'inscrit. C'est le cas par exemple des études sur l'aménagement participatif au Brésil, qui ont mis l'accent sur la formation et le rôle des leaders au sein des populations pauvres. La thèse de Debora Nunes ${ }^{4}$, sur l'aménagement participatif dans un quartier populaire de Salvador est tout à fait intéressante sur ce plan. L'analyse qu'elle fait du comportement du leader, des raisons qui l'ont conduit à une position de pouvoir au sein du groupe, (qui est une analyse de type anthropologique), met en effet en lumière deux aspects du problème: d'une part, la situation de dépendance dans laquelle se trouvent ces populations par rapport à des intermédiaires dont elles ont besoin pour dialoguer avec les pouvoirs, - les savants, comme ils disent - , qui est liée à leur position sociale et à la façon dont ils se perçoivent dans la société, (avec en arrière plan leur manque de confiance, une certaine honte d'être pauvre, etc.) ; d'autre part, les véritables aspirations et intentions du leader lui-même, qui la plupart du temps va trahir son groupe, et utiliser son pouvoir pour se faire acheter par "les politiques", 
c'est-à-dire par des politiques qui cherchent à «récupérer » les pauvres. Dans cet exemple, on voit que les deux démarches, - l'une au niveau du groupe dans son contexte, l'autre au niveau de l'individu - sont indispensables, à la fois pour comprendre comment fonctionne l'aménagement participatif et pour aller plus loin dans la compréhension des limites de la participation populaire dans la réorganisation des espaces de pauvreté.

21 D'après ce que tu dis, on a l'impression que, la recherche urbaine dans les pays en voie de développement est en avance par rapport à celle sur nos pays. En France il y a des géographes qui travaillent dans une perspective similaire, - sur les banlieues par exemple -, mais on peut se demander si les anthropologues et les sociologues ne se sont beaucoup plus emparés de ces problèmes que les géographes. Quel peut être selon toi l'apport spécifique des géographes sur des problèmes comme l'exclusion, la ségrégation, la ghettoïsation, la violence urbaine etc. ?

22 Je pense en effet qu'il y a eu une évolution extrêmement positive de la démarche urbaine sur les pays du Sud depuis une quinzaine d'années, et plus particulièrement dans ceux que je connais bien, le Brésil et les pays du Maghreb, et cela n'est pas le fait de géographes uniquement. J'ai été très impressionné il y a une dizaine d'années déjà par la richesse de la thèse d'Alain Dubresson sur la Côte d'Ivoire, et je le suis actuellement par l'évolution des études urbaines au Brésil. Ce sont ces travaux qui m'ont le plus appris. Cela est sans doute lié au fait que dans les pays en développement les inégalités sociales sont plus fortes et que leurs implications spatiales sont plus criantes. Les chercheurs brésiliens qui travaillent sur les questions urbaines dans les directions que je viens d'évoquer sont aussi pratiquement tous des gens qui, d'où qu'ils viennent, ont une idée des luttes sociales ou sont engagés dans les luttes sociales, ce qui explique qu'ils essaient d'aller jusqu'au bout. Sur la France, je n'ai pas une vue assez globale des travaux récents, même sur les banlieues, pour porter un jugement, mais il est vrai que je n'ai pas rencontré d'études aussi probantes que celle que je viens d'évoquer par exemple sur l'Afrique du sud. Il y a des géographes qui ont avancé dans des directions similaires, avec une approche plus anthropologique. C'est le cas par exemple de Hervé Vieillard-Baron, dont les travaux m'ont beaucoup intéressé mais que d'autres ont beaucoup critiqués, ou de ceux qui ont mis récemment l'accent sur la différence entre exclusion sociale et exclusion spatiale, et montré qu'il n'y avait pas nécessairement adéquation entre l'un et l'autre. Il y a là me semble-t-il des apports intéressants, car le problème de l'exclusion est envisagé sous l'angle des rapports société/espace. Mais ceci suppose de faire porter l'effort jusqu'à l'analyse des comportements individuels. Or, les géographes demeurent encore très marqués par une approche globale des problèmes urbains. Il me semble qu'ils ont pourtant beaucoup à apporter, en poussant plus loin l'analyse des dimensions spatiales des problèmes sociaux.

Sur la violence urbaine, par exemple, il ne suffit pas de constater qu'elle vient des jeunes et qu'ils sont violents parce qu'on ne leur donne pas d'autres alternatives. Les géographes peuvent certainement aller plus loin dans la compréhension du phénomène et de ses manifestations variables selon les lieux, d'une part, en revenant sur l'interrelation entre la dégradation d'un groupe social pour des raisons socioéconomiques, et la dégradation de son espace pour des raisons de vieillissement ou d'inadaptation des conditions d'habitat et du cadre de vie; d'autre part, en prolongeant cette analyse par une étude plus fine au niveau des comportements individuels. Il y a des révoltes qui sont plus ou moins liées au contexte général, et d'autres dont les 
raisons sont plus liées à l'histoire individuelle et familiale. Si j'avais à diriger des études sur la violence urbaine, qui a sa place dans un questionnement géographique, c'est dans ce sens que je les orienterais. La question de l'environnement urbain mériterait aussi à mon sens un effort de réflexion plus poussé, car il y a aujourd'hui beaucoup d'études sur la dégradation des conditions de vie et de l'environnement, mais l'environnement constitue un tel enjeu politique, qu'il y a beaucoup de perversion dans les approches qui en sont faites, que les problèmes soient minimisés ou exagérés. Il faudrait donc que des chercheurs travaillent sur l'environnement avec un peu plus de recul par rapport aux enjeux politiques.

24 Est-ce que tu penses que les jeunes chercheurs peuvent vraiment arriver à tenir la réalité, les vrais problèmes, par les deux bouts, c'est-à-dire à la fois par des approches plus micro, sur des sujets plus pointus, et par des approches plus globalisantes, plus macro?

Il y aura toujours des chercheurs qui insisteront plus sur une dimension ou un aspect de la réalité que sur les autres. Mais quelle que soit la question abordée, je pense que de plus en plus on ne peut faire l'impasse de l'analyse au niveau de l'acteur individuel, à condition de respecter l'interaction des éléments en jeu à différents niveaux et/ou différentes échelles. Sur les processus et formes de péri-urbanisation dans la région parisienne, par exemple, on ne peut plus s'en tenir à des analyses sur la spéculation immobilière et foncière. On a déjà beaucoup d'études sur le sujet. Si l'on veut aller plus loin, il faudra sans doute creuser la question de la dynamique interne des conseils municipaux et du rôle des maires, car c'est en partie d'eux que dépendent les autorisations de construction, et leurs comportements ne relèvent pas seulement de majorités politiques différentes. C'est un aspect de la question que Monique Roussel ${ }^{5}$ avait esquissé dans sa thèse. Il y a des mécanismes qui échappent aux maires, mais ce sont leurs capacités d'action et de pression variables pour faire accepter des projets spéculatifs qui vont contribuer à nuancer le phénomène selon les lieux, et cela n'est pas dissociable de leur position sociale et des rapports qu'ils entretiennent avec la population, que ceux-ci soient d'ordre matériel ou idéologique. Au stade actuel de la recherche urbaine, et si l'on replace bien l'intervention de l'acteur individuel dans le contexte socio-économique plus global et les relations sociales dans laquelle elle s'inscrit, c'est dans ce sens qu'il faut orienter la recherche. Il ne faut pas opposer le micro et le macro. Il faut s'efforcer au contraire de manier les niveaux d'analyse, même si cela n'est pas toujours évident.

Inversement quand on revient sur l'analyse du contexte socio-économique ou de processus plus globaux, il y a beaucoup de géographes qui travaillent sur les conditions d'adaptation des entités locales aux restructurations de l'économie, sur leurs capacités d'innovation et de changement. À ton avis, qu'est ce que les géographes peuvent apporter de spécifique sur cette question? Par quel biais aborder la question?

On peut envisager la question du développement local sous deux angles principaux. D'abord sous l'angle de l'action. Il s'agit dans ce cas de tirer le meilleur parti des conditions locales ou de les valoriser pour attirer des investissements susceptibles de générer l'innovation et de fixer à un endroit des éléments de croissance économique. À ce niveau, le développement local dépend donc principalement de la capacité des hommes politiques locaux à dialoguer et négocier avec les forces économiques privées et avec les grands décideurs. C'est ce qui va permettre d'orienter la stratégie des investisseurs vers telle ville plutôt que telle autre. Il s'agit donc de comprendre des 
stratégies économiques plus globales, ce qui relève beaucoup plus à mon sens d'une analyse économique.

La question du développement local renvoie par ailleurs aux inégales capacités d'autodéveloppement de la population et des entreprises locales. Dans les villes d'Afrique Noire on peut ainsi penser, à condition d'être optimiste, que le secteur informel est susceptible de devenir un élément important de la croissance. Mais dans ce domaine, ce que peuvent apporter de spécifique les géographes par rapport aux anthropologues et aux économistes n'est pas évident, sauf à arriver à analyser comme l'a fait Pascale Froment sur Naples, la dynamique profonde des rapports qui unissent une société à un territoire, et qui explique les capacités d'émergence de nouveaux entrepreneurs à un endroit plutôt qu'à un autre. On en revient à l'histoire de la bourgeoisie lyonnaise, qui explique qu'elle soit plus performante actuellement dans ses dialogues avec les grands investisseurs pour influencer leurs stratégies territoriales que la bourgeoisie de Toulouse ou de Montpellier.

Comment expliques-tu alors que les géographes soient de plus en plus pris au sérieux par les économistes? Ton apport au groupe de synthèse Prospective et Territoires de la Datar a été, on le sait, très apprécié, alors que vous n'étiez que deux géographes dans ce groupe. À la lumière de tes expériences, quel peut être le rôle des géographes en matière d'aménagement?

Si les géographes sont de plus en plus pris au sérieux et ont un rôle de plus en plus important au niveau de l'aménagement régional, c'est parce qu'ils envisagent les problèmes au regard d'une analyse qui met en jeu des interdépendances multiples entre l'économique et le social en les raccrochant bien aux spécificités territoriales. Les géographes ont donc un rôle à jouer dans de nombreux domaines, y compris celui du développement local. Mais à ce niveau, ce sont les stratégies des investisseurs et des décideurs qui sont déterminantes et le rôle des géographes, en tant que scientifiques, est nécessairement limité. Ils peuvent faire valoir auprès des investisseurs les avantages de la ville, les atouts des territoires, même si cela est un peu en marge d'une démarche scientifique. Mais ce qui va l'emporter, ce sont les avantages financiers que les grands investisseurs vont pouvoir retirer du lieu.

31 La question se pose différemment lorsqu'il s'agit d'expliquer les capacités variables de "réussite» selon les lieux, et non d'agir au futur. Quand il s'agit de recherche, les dimensions politiques du problème et les stratégies des acteurs peuvent être prises en compte. Il reste cependant que ce n'est pas toujours en raison des avantages qu'offre un territoire, que les investisseurs vont s'y implanter. Quand les Japonais décident de s'implanter à Valenciennes, je ne suis pas sûr que, s'ils nous avaient demandé de leur faire une étude de localisation, on leur aurait dit de s'implanter là.

\section{NOTES}

1.Cf. Jean Copans et Roger Meunier (dir.) «Afrique du Sud : les débuts de la transition », Tiers-Monde, $\mathrm{n}^{\circ}$ 159, juillet/septembre 1999. 
2.Pascale Froment, Économie informelle et Italie du Sud; le cas du secteur des cuirs et chaussures à Naples, thèse de doctorat, sous la direction de Michel Rochefort, université de Paris I, 1999.

3.Voir dans ce volume, l'entretien avec Dominique Rivière « Michel Rochefort et l'aménagement ".

4.Debora Nunes, L'apprentissage de la citoyenneté à partir du travail communautaire. L'exemple d'un quartier populaire de Salvador, Brésil. thèse de doctorat, IUP, université de Paris 12-Val de Marne, 1998.

5.Monique Roussel, « Les marges de l'urbanisation : formes et processus de périurbanisation ", thèse de troisième cycle, sous la direction de J. Bonnamour, université de Paris I, 1987.

\section{AUTEURS}

JACQUES BRUN

Professeur à l'université de Paris 1 , Ladyss

CATHERINE PAIX

Ladyss, Cnrs 